НЗВЕСТИЯ АКАДЕМИИ НАУК ЭСТОНСКОИ ССР. ТОМ 28 ФИЗИКА * МАТЕМАТИКА. 1979, № 1

\title{
МНОГОЦЕЛЕВОЙ МЕТОД РАСПРЕДЕЛЕНИЯ ЭФФЕКТИВНЫХ ВИДОВ ТОПЛИВА МЕЖДУ РАЙОНАМИ В УСЛОВИЯХ ВЕРОЯТНОСТНОГО ХАРАКТЕРА ИСХОДНОЙ ИНФОРМАЦИИ
}

K. JAANIMAGI. MITME-EESMARGILINE EFEKTIIVSETE KOTUSELIIKIDE RAJOONIDEVAHELISE JAOTAMISE MEETOD TOENATOSE LAHTEINFORMATSIOONI KORRAL

K. JAANIMÁGI. MULTICRITERIAL DISTRIBUTION METHOD OF THE EFFECTIVE KINDS OF FUEL IN CONDITIONS OF PROBABILISTIC INITIAL INFORMATION

\section{(Представлена И. Эпиком)}

Пусть для оптимизации топливно-энергетического баланса (ТЭБ) объединения районов построены обобщенные энергоэкономические характеристики (ОЭЭХ) [ $\left.{ }^{1}\right]$, учитывающие наряду с многокритериальностью функционирования энергетических систем и случайность исходной информации. ОЭЭХ в простейшем случае представляет собой полином второго порядка:

$$
Z_{u}=a_{0 u}+\sum_{i=1}^{n} a_{i u} x_{i u}+\frac{1}{2} \sum_{i=1}^{n} a_{i i u} x_{i u}^{2}+\sum_{i, j=1}^{n} \sum_{i<j} a_{i j u} x_{i u} x_{j u},
$$

где $u=1,2, \ldots, U-$ районы, для которых построены ОЭЭХ ТЭБ; $Z_{u}$ - обобщенный критерий в математической модели ТЭБ района $\left[{ }^{1}\right]$; $x_{i u}, x_{j u}$ - количества $i$-го и $j$-го видов топлива в ТЭБ $u$-го района; $a_{0 u}, a_{i u}, a_{i i u}, a_{i j u}$ - случайные коэффициенты, моделируемые согласно методу, изложенному в $\left[{ }^{2}\right]$.

Для любого $u$, в силу положительной определенности матрицы $\left\{E a_{i j}\right\}$, $E Z_{u}=E a_{0 u}+\sum_{i=1}^{n} E a_{i u} x_{i u}+\frac{1}{2} \sum_{i=1}^{n} E a_{i i u} x_{i u}^{2}+\sum_{\substack{i=1 \\ j=1}}^{n} \sum_{i<j} E a_{i j u} x_{i 4} x_{j 4}-$ выпуклые функции.

Для определения оптимальных количеств эффективных видов топлива в районе реша'ется задача

$$
\begin{aligned}
E\left(Z_{u}\right) \rightarrow \min , \quad u & =1,2, \ldots, U, \\
\sum_{u=1}^{U} x_{i u} & =X_{i} .
\end{aligned}
$$

Пусть $E Z_{u}$ ид - оптимальное значение целевой функции $E Z_{u}$ на области (3). Назовем вектор $\left(E Z_{1}^{\text {шд }}, E Z_{2}^{\text {шд }}, \ldots, E Z_{u}\right.$ ид) идеальным решением задачи (2), (3).

Первый метод решения задачи векторной оптимизации (2), (3) подразумевает построение множества Парето на основе известной леммы 
Карлина [ $\left.{ }^{3}\right]$ с последующим выделением из этого множества плана, наименее удаленного от идеального решения задачи (2), (3). Для нахождения множества Парето при различных $\lambda_{u} \geqslant 0, \sum_{u=1}^{U} \lambda_{u}=1$ решается задача квадратичного программирования

$$
\Phi(\bar{X})=\sum_{u=1}^{U} \lambda_{u} E Z_{u}(\bar{X}) \rightarrow \min
$$

при $\bar{X}=\left\{x_{i u}\right\} \quad(i=1,2, \ldots, n ; u=1,2, \ldots, U)$, удовлетворяющем равенству (3). Для выбора окончательного решения из множества Парето, а значит, и для задания весовых коэффициентов $\lambda$ таких, при которых получается оптимальный план задачи (2), (3), может быть применен метод экспертных оценок.

Второй метод решения задачи (2), (3) основан на сравнении различных допустимых ее решений. Примем, что допустимый план $\bar{X}_{1}$ предпочтительнее плана $\bar{X}_{2}\left(\bar{X}_{1}<\bar{X}_{2}\right)$, если выполняется соотношение

$$
\sum_{u=1}^{U}\left(E Z_{u}\left(\bar{X}_{1}\right)-E Z_{u}^{\text {onT }}\right)<\sum_{u=1}^{U}\left(E Z_{u}\left(\bar{X}_{2}\right)-E Z_{u}^{\text {orr }}\right)
$$

Отметим, что в процессе сравнения допустимых решений по отношению предпочтительности лицо, принимающее решение, может вносить определенные коррективы в этот процесс. Допустим, что нами получено

$$
\bar{X}_{1}<\bar{X}_{2}<\bar{X}_{3}<\ldots<\bar{X}_{k} .
$$

За оптимальный компромиссный план задачи (2), (3) принимаем план, минимизирующий функционал (4) на области (3). Из условия (5) следует, что для нахождения весовых коэффициентов, фигурирующих в (4) и учитывающих значимость отдельных критериев (2), необходимо решить систему неравенств

$$
\Phi\left(\bar{X}_{1}\right)<\Phi\left(\bar{X}_{2}\right)<\ldots<\Phi\left(\bar{X}_{k}\right)
$$

при условии

$$
\sum_{u=1}^{U} \lambda_{u}=1, \quad \lambda_{u} \geqslant 0
$$

Проанализировав энергетические системы всех рассматриваемых в задаче (2), (3) районов, можно наложить на коэффициенты $\lambda$ дополнительные условия вида $\lambda_{i}>\lambda_{j}, j \neq i$. Для выбора окончательного реше-

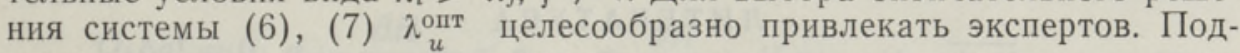
ставляя $\lambda_{u}^{\text {oпт }}$ в функционал $\sum_{u=1}^{U} \lambda_{u}^{\text {oпr }} E Z_{u}(\bar{X})$ и минимизируя его на области (3), получим решение задачи (2), (3). Из определения оптимума Парето следует, что предложенная схема решения не выводит нас за рамки множества Парето задачи (2), (3).

Рассмотрим численный пример из [ $\left.{ }^{4}\right]$. Имеются четыре района, использующие три вида топлива (см. таблицу).

Решение задачи квадратичного программирования (2), (3) первым методом было реализовано на ЭВМ ЕС-1022 с помощью специальной программы, базирующейся на применении метода множителей Лагранжа (5000 раз при различных $\lambda_{i}$, равномерно распределенных на отрезке $(0,1))$. В качестве оптимального компромиссного решения была получена точка Парето $\bar{X}=\left(x_{11}, x_{12}, x_{13}, x_{14}, x_{21}, x_{22}, x_{23}, x_{32}, x_{33}, x_{34}\right)=$ $=(6,56 ; 4,04 ; 2,9 ; 0,9 ;-2,64 ; 1,90 ; 6,43 ;-0,33 ;-0,39 ; 12,87)$ с $\lambda_{1}=$ 


\title{
Коэффициенты ОЭЭХ районов
}

\begin{tabular}{|c|c|c|c|c|}
\hline & I район & II район & III район & IV район \\
\hline $\begin{array}{l}E a_{11} \\
E a_{22} \\
E a_{33} \\
E a_{12} \\
E a_{13} \\
E a_{23} \\
E a_{1} \\
E a_{2} \\
E a_{3} \\
E a_{0}\end{array}$ & $\begin{array}{r}4,6 \\
5,1 \\
\overline{4,0} \\
\overline{-} \\
-2 \overline{4,4} \\
-18,0 \\
2 \overline{7} \overline{7,3}\end{array}$ & $\begin{array}{r}8,5 \\
9,2 \\
9,5 \\
6,4 \\
6,7 \\
8,5 \\
-48,4 \\
-42,4 \\
-42,6 \\
318,4\end{array}$ & $\begin{array}{r}2,2 \\
4,8 \\
4,8 \\
2,6 \\
2,6 \\
4,4 \\
-26,9 \\
-40,2 \\
-39,2 \\
402,8\end{array}$ & $\begin{array}{c}0,26 \\
\overline{0,53} \\
\overline{0,24} \\
-\overline{-}, 59 \\
-10,1 \\
689,3\end{array}$ \\
\hline & $=14,4$ & $X_{2}=$ & & $=12,15$ \\
\hline
\end{tabular}

$=0,13, \lambda_{2}=0,38, \lambda_{3}=0,18, \lambda_{4}=0,31$. Экономический эффект, подсчитанный по методике [ $\left.{ }^{4}\right]$, составил 11,47 млн. руб. в год.

Применение второго метода решения дало оптимальный компромиссный план $\bar{X}=(6,6 ; 3,58 ; 4,28 ;-0,05 ;-2,35 ; 2,30 ; 5,74 ;-0,54 ;-0,14$; 12,83) с $\lambda_{1}=0,194, \lambda_{2}=0,23, \lambda_{3}=0,285, \lambda_{4}=0,291$. Экономический эффект составил 9,57 млн. руб. в год.

Отметим, что решать задачи большой размерности проще с помощью второго метода, хотя ошибка, вносимая в расчеты, при этом более значительна.

Итак, к достоинствам многоцелевого метода распределения эффективных видов топлива между районами можно отнести следующее.

1. Его относительная простота по сравнению с методами, основанными на решении задач линейного программирования.

2. Возможность учета в ТЭБ как имманентных интересов каждого района, так и эмерджентных интересов объединения районов путем привлечения подлежащего минимизации критерия математического ожидания ОЭЭХ, коэффициенты которого рассчитываются по методике $\left[{ }^{2}\right]$. 3. Возможность использования в качестве ОЭЭХ района модели более сложного вида, нежели полином второго порядка, что повышает точность аппроксимации $Z_{u}$.

4. Возможность учета многокритериальности развития энергетических систем каждого района.

\author{
Л И ТЕ Р А Т У РА
}

1. Я а н и яги К. Э., В сб.: Учет неопределенности исходной информации при оптимизации энергетического хозяйства экономнческого района, Таллин, Изд. АН ЭССР, 1978, c. 159-163.

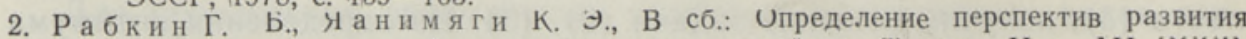
энергетического хозяйства экономического района, Таллин, Изд. АН ЭССР, 1977 , c. $159-162$.

3. К арлин С., Математические методы в теории игр, программировании и экономике, М., «Мир», 1964.

4. В а йк Л. Э., Принципы и методы взаимосвязанной оптимизации топливно-энергетического хозяйства районов и страны, Автореф. докт. дисс., М., 1976.

Институт термофизики и электрофизики Академии наук Эстонской ССР

Поступила в редакцию 30/VI 1978 